\title{
Timing of do-not-resuscitate order and aggressiveness of care near the end of life in cancer patients: a retrospective cohort study
}

\author{
Panpan Cui ${ }^{1}$, Shiqi Dong ${ }^{1}$, Xinyi Gao ${ }^{1}$, Yiyang Chen ${ }^{1}$, Han Tang ${ }^{1}$, Changying Chen ${ }^{1}, 2^{*}$ \\ 1 Nursing College of Zhengzhou University, Henan, China \\ 2 The First Affiliated Hospital of Zhengzhou University, Henan, China \\ * Corresponding author
}

\section{Introduction}

Patients with advanced cancer are often cared for and die in hospitals in China. Do-not-resuscitate (DNR) order is crucial in end-of-life care for them. This study examined the association between the timing of DNR orders and the aggressiveness of end-of-life care.

\section{Objectives}

The aims of this study were to:

1. Describe the timing of signing DNR orders,

2. Explore the association between signing a DNR order and health care utilization during the last hospitalization among advanced cancer patients in mainland China.

\section{Methods}

A retrospective chart review was done in three tertiary hospitals in China. Data about cancer patients' characteristics, DNR order, aggressive and comfort measures were collected during the last hospitalization since January 2016 to December 2017.

Measurement. A data extraction form was developed by the research team according to the framework of the research (Figure 1). It was composed of:

(1) Timing of DNR orders;

(2) Health care utilization (comfort measures and life-sustaining treatments (LSTs)).

\section{Figure 1 Framework of research}

Framework of Research

Advance Directives

DNR order

- Timing (within or beyond 3 days before death)
Health Care Utilization

Comfort medication

- Sedatives

- Analgesics

- Psychological support

Life-Sustaining Treatments

- ICU admission

- Aggressive procedures (CPR, intubation, mechanical ventilation, chemotherapy, dialysis, blood transfusion, nasal feeding, etc)

\section{Results}

- 252 patients were included in the analysis. The median time intervals between signing the DNR order and patient's death was 1 day (range: $1-107$ days), and $54.4 \%$ were signed within three days before death (Figure 2 ).
- Compared with patients who signed a DNR order within 3 days, those who signed beyond 3 days were less likely to transfer to ICU, undergo tracheal intubation, ventilation, dialysis, blood transfusion and CPR, while more likely to be given morphine and psychological support (Table 1).

Figure 2 Interval between signing DNR orders and patient death

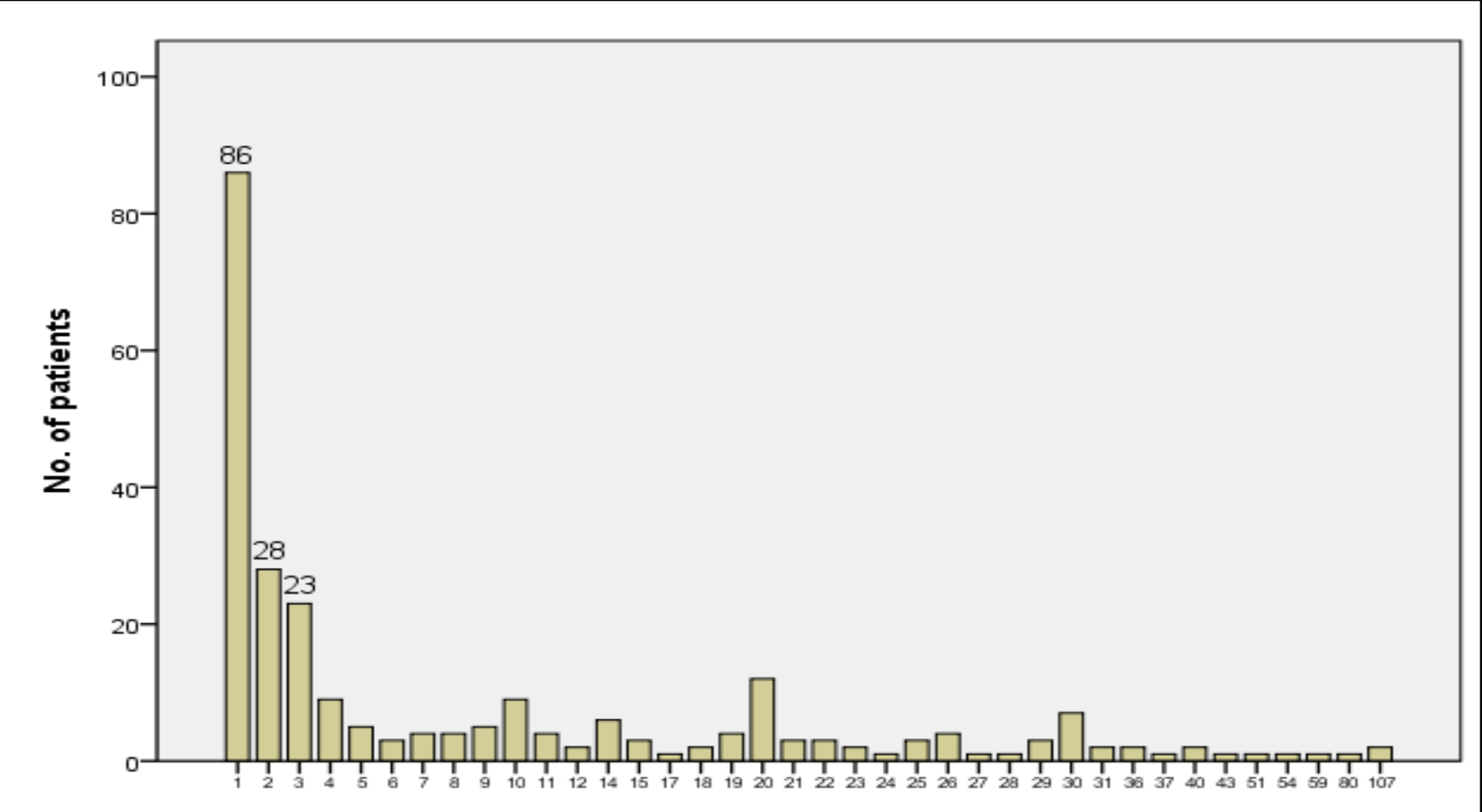

Table 1 Likelihood of receiving health care utilization according to the timing of DNR orders

\begin{tabular}{lll}
\hline & OR $(95 \% \mathrm{Cl})$ & $P$ value \\
\hline Aggressive procedures & & \\
ICU admission & $0.295(0.137-0.638)$ & $\mathbf{0 . 0 0 2}$ \\
CPR & $0.090(0.047-0.173)$ & $\mathbf{0 . 0 0 0}$ \\
Ventilation & $0.186(0.066-0.521)$ & $\mathbf{0 . 0 0 1}$ \\
Dialysis & $0.171(0.062-0.476)$ & $\mathbf{0 . 0 0 1}$ \\
Chemotherapy & $0.855(0.355-2.059)$ & 0.727 \\
Blood transfusion & $0.439(0.237-0.815)$ & $\mathbf{0 . 0 0 9}$ \\
Tracheal intubation & $0.293(0.116-0.741)$ & $\mathbf{0 . 0 1 0}$ \\
Comfort measures & & \\
Sedatives & $1.303(0.766-2.217)$ & 0.329 \\
Morphine use & $3.103(1.773-5.431)$ & $\mathbf{0 . 0 0 0}$ \\
Psychological support & $2.540(1.469-4.391)$ & $\mathbf{0 . 0 0 1}$ \\
\hline
\end{tabular}

${ }^{*}$ Reference group: patients who signed DNR orders within 3 days before death

\section{Conclusions}

We found that DNR orders were always signed late. Cancer patients who signed DNR orders beyond 3 days before death were less likely to undergo aggressive procedures but more likely to be given comfort measures, compared with those within 3 days. Further studies are necessary to better understand the optimum timing and promote early DNR orders in advanced cancer patients.

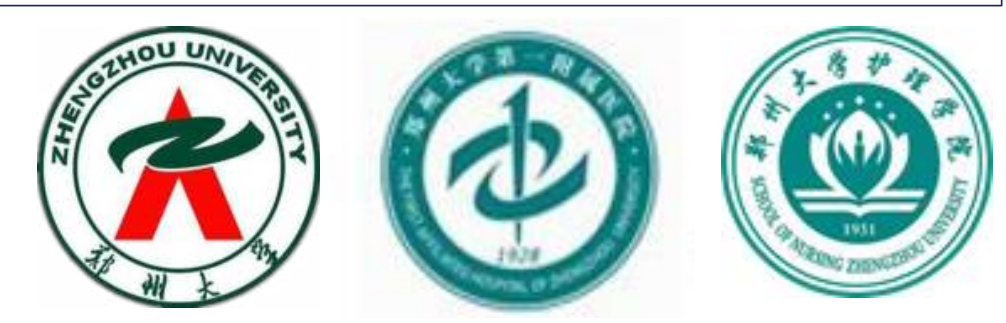

